\title{
Künstliche Intelligenz der Messgeräte
}

\author{
József Kohut
}

Die Intelligenz der Messgeräte wird grundsätzlich durch ihr Verhältnis zu den Computern bestimmt. Geschichtlich gesehen lassen sich im Gebiet der Technik vier Perioden der Computer-Mesgerät-Kopplungen unterscheiden: vom Anfang an bis zum Jahre 1972 sprechen wir über die Periode des „Getrenntseins“, ab 1972 (also von der Erscheinung der GPIB Schnittstelle-Norm) über die Periode der „Kooperation“, ab 1980 uiber die Periode der „Einbettung“ (gekennzeichnet von den in den Messgeräten eingebetteten Mikroprozessoren und von den im Computer eingebetteten Meßgeräten), und im Jahre 2000 hat die Periode der Computernetze begonnen (die Verknüpfung der Messgeräte mit LAN und WAN).

Die Definition und die meisten traditionellen Faktoren der menschlichen Intelligenz sind auch auf die künstliche Intelligenz der Messgeräte zu übertragen. In gewisser Hinsicht hat der Computer der menschlichen Intelligenz gegenüber einen Vorteil, in anderen Gebieten aber befinden sich die Computer (und die mit dem Computer immer enger verbundenen Messgeräte) im erheblichen Nachteil.

\section{Die menschliche Intelligenz}

Der Erfolg und die Wirksamkeit des Verhältnisses des Menschen zu seinen Mitmenschen und seiner Umgebung hängt sehr stark von der Intelligenz des jeweiligen Menschen ab. Es gab sehr viele Versuche, den Begriff der Intelligenz in unterschiedlichen Formen zu definieren. Ein Teil der Definitionen ist zu allgemein, in einem anderen Teil werden gewisse für die Intelligenz typische Dinge betont, trotzdem ist aber dieser Teil nicht umfangreich genug.

Die Definition von Wechsler aus dem Jahre 1939 klingt so: „Intelligenz heißt eine globale Fähigkeit des Menschen, die einem ermöglicht, zielorientiert zu handeln, rationell zu denken und erfolgreich mit der Umgebung umzugehen." Diese Definition ist einerseits geeignet, um den Sinn der Intelligenz zu definieren, andererseits gibt sie aber nicht genügend Richtlinien zu weiterer Untersuchung des Begriffes.

Ungefähr in dieser Zeit (1938) hat Thurstone die Faktoren der menschlichen Intelligenz so definiert, dass man eine Hilfe bekommt sich zurechtzufinden.

Diese Faktoren sind:

- das sprachliche Verstehen (also die Verwendung des sogenannten zweiten Signalsystems in der schriftlichen und mündlichen Kommunikation),

- die Schnelle des Findens der Wörter (die Geschwindigkeit der Entgegnung im Laufe des Sprechens),

- die Fähigkeit des Rechnens (und im Zusammenhang damit die abstrakte Denkweise),
- die Wahrnehmung der räumlichen Relationen (durch das Sehvermögen und das Gehör, mit der Hilfe des Tastsinnes bzw. mit der Anwendung der Erinnerung und durch Folgerung),

- die Wahrnehmung der Relationen in der Umgebung (die Identifizierung der Form, der Temperatur, des Duftes, des Geschmackes, der Bewegung, der Qualität der Oberfläche etc. von unterschiedlichen Gegenständen),

- das Gedächtnis (die Reproduktion von früheren Ereignissen, Gefühlen und deren zeitlichen Kontakten),

- die Folgerung (die Erkennung einer logischen Verbindung in den Vorgängen, und aufgrund dieser Erkennung eine erfolgreiche Definition bzw. Prognostizierung von nicht bekannten Vorgängen in der Vergangenheit oder in der Zukunft).

Die Größe der menschlichen Intelligenz ist nicht gleich der Summe der Größen der einzelnen Faktoren, sondern wird von der Verkettung, Proportionalität dieser Faktoren und sogar von der menschlichen Motivation, Selbstbeherrschung, Laune beeinflusst.

\section{Periode und charakteristische Eigenschaften der "Messgerät-Rechner-Kopplung"}

Was die Kopplung der Messgeräte mit den Rechnern betrifft, lassen sich vier Periode unterscheiden. In den einzelnen Perioden ist immer eine kontinuierliche technische Entwicklung zu beobachten, aber jede einzelne Periode weist im Ganzen ein einheitliches Bild auf. Der fachliche Inhalt dieser Periode lässt sich abhängig von der Entwicklungsstufe der Hardware, des Computerprogramms, des Messgeräts und der Messmethode einzeln untersuchen.

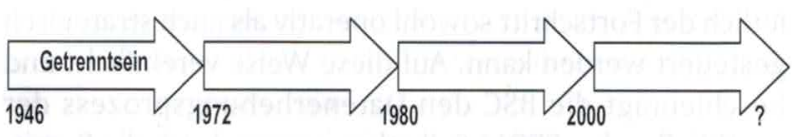

Die Periode des Getrenntseins dauerte von der ersten Generation der Rechner etwa bis zum Jahre 1972. In dieser Periode waren die Rechner sehr große, kostbare Geräte mit großem Energieverbrauch. Trotz dieser Eigenschaften konnten diese Geräte im Vergleich zu den heutigen Computern relativ wenige Operationen durchfuihren, wenige Daten speichern und ihre Geschwindigkeit war auch ziemlich niedrig (obwohl schon die Computer der ersten Generation fähig waren, die rechnerischen Operationen wesentlich schneller als der Mensch durchzufuihren). Die Computer waren in den Rechenzentren aufgestellt, sowohl die Anschaffungs- als auch die 
Betriebskosten waren sehr hoch und oft wurden sie zu militärischen Zwecken verwendet. Deswegen kam ein unmittelbarer Kontakt mit den Messgeräten damals gar nicht in die Frage. Das Programmieren der Computer wurde meistens mit der Anwendung sehr einfacher Maschinencodes, oder symbolischen Codes durchgeführt. Die ausgestalteten höhere Programmiersprachen waren abhängig von den Computertypen und von den unterschiedlichen Anwendungsgebieten, die Frage ihrer Generalisierung hat sich damals noch nicht gestellt. In jener Zeit war der logische Aufbau der Messgeräte sehr einfach, ihre Bedienungseinheiten, Anzeiger, Signalgeneratoren und Auswertungseinheiten funktionierten damals in analoger Weise. Es lohnt sich schon hier zu bemerken, dass modulare Messgeräte nur unter speziellen Bedingungen angewendet wurden (NIM-System für isotoptechnische/nukleare Versuche). Die Steuerung des Messprozesses, das Bedienen der Messinstrumente hat natürlich auch menschliche Mithilfe beansprucht, die Dokumentation der Ergebnisse und ihre Bearbeitung erfolgte mit der „Papier-Bleistift-Methode“, manchmal mit der Hilfe von einem mechanischen Rechner oder Rechenschieber oder mit der Off-Line-Nachbearbeitung der in ein Rechenzentrum geschickten Daten.

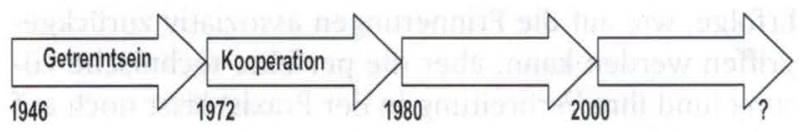

Die Periode der Kooperation rechnet man beginnend mit dem Erscheinen der den Rechner und das Messgerät zusammenkoppelnden Schnittstelle. Aus der am Anfang chaotischen Entwicklung hat sich die standardisierte Schnittstelle HP-IB (auch als GPIB, IEC-625, IEEE 488 bekannt) im Jahre 1972 entwickelt. Diese Schnittstelle wird auch heute massenhaft angewendet, sogar in einer in vieler Hinsicht weiterentwickelten Form bildet sie die Grundlage anderer Schnittstellen-Normen (IEE 488.2, SCPI, VXI, PXI). In der Periode bis 1980 ist der Computer - Dank der Entwicklung der Technik der integrierten Schaltkreise - ein Gerät geworden, dessen Körperinhalt, Energieverbrauch und Preis mit denen der Messgeräte zu vergleichen sind. Die Gestaltung des Tischrechners hat die alltägliche Arbeit mit dem Computer erleichtert. Die die Operationen durchführenden Fähigkeiten sind sehr stark angestiegen, die Datenspeicher und die Programmspeicher sind schneller geworden, wobei ihre Kapazität auch größer geworden ist. Die gestaltenden höheren Programmiersprachen (COBOL, FORTRAN, ALGOL60) waren meistens mathematische Konstruktionen, bei denen nur ein Teil ihrer algorithmischen Möglichkeiten mit Hilfe eines Compilers für den Rechner übersetzt werden konnte. Schon in dieser Zeit wurden die Grundlagen der multitask Programmdurchführung, d. h. die der Konkurrenz-Programmierung festgelegt. Die Messgeräte sind mehr und mehr digitalisiert worden (Bedienungseinheit, Anzeiger, innere Datenspeicherung, logische Entscheidungen), und die Fernbedienung ist eine mögliche Option der Geräte geworden. Die modularen Messinstrumente (CAMAC) sind in immer mehr Anwendungen erschienen und das Netz der Hersteller von kompatiblen Modulen erweiterte sich weltweit. Der
Messprozess hat sich umgewandelt, das Messprogramm (die Steuerung der Instrumente, die Bearbeitung und die Dokumentierung der Ergebnisse) wurde von einem externen Rechner durchgefuihrt.

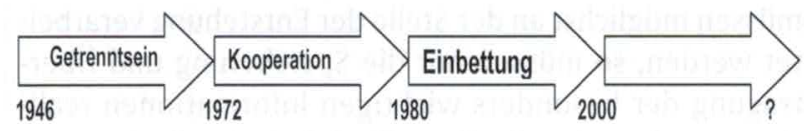

Die Periode der Einbettung. Die Entwicklung der LSIVLSI Technik hat ungefähr vom Jahre 1980 an die Verbreitung der Mikroprozessoren ermöglicht. Mit der Erscheinung der IBM-Personalcomputers sind die Rechner sehr schnell billiger werdende Geräte geworden, die uiberall in großer Auswahl zu kaufen sind, wobei die Operationsgeschwindigkeit und die Datenspeicherungskapazität der Geräte sprunghaft angewachsen ist. Die Anwendung der Rechner in der Industrie und in der Messtechnik ist ein organischer Teil der technischen Praxis geworden. Es haben sich die Systeme der modulär aufgebauten Rechner herausgebildet, von denen sich die VME-Norm am meisten verbreitet hat. Weiterhin haben sich die allgemein verwendbaren Programmiersprachen und Operationssysteme herausgebildet, womit der Routineteil der Arbeit der Programmentwickler vereinfacht wurde. Es wurden spezielle Methode entwickelt, um in der Anfertigung der algorithmischen Teile von Programmen eine Hilfe leisten zu können. Mit der Hilfe der objektorientierten Programmierung, der visuellen und der grafischen Programmierung werden die Probleme unterschiedlicher Gebiete der Programmierung gelöst.

Die große Speicherkapazität und schnelle Durchführung der Operationen ermöglichen die Gestaltung von Expertensystemen, die mit der Zusammenordnung der Kenntnisse in gewissen Fachgebieten sogar zur Beratung anzuwenden sind. Der Rechner ist als Bauelement ins Messgerät eingebettet. Die Mikrocontroller und die Signalprozessoren haben immer mehr von den Innenfunktionen übernommen, und mit ihrer software-ähnlichen Funktionsweise haben sie die Menge der Hardware auf ein Minimum vermindert. Ein ins Messgerät eingebettete Rechner (manchmal sogar mehrere Rechner) ermöglicht dem Messgerät - ohne einen externen Rechner zu haben - zusammengesetzte Funktionen zu realisieren, hochentwickelte Datenvorbereitungen und Auswertungen durchzufuihren. Es sind solche Dinge ermöglicht worden, wie z. B. Selbsttest der Funktionen der Geräte, das metrologische Selbstkalibrieren und Erzielung genauerer Ergebnisse mit der Anwendung der Korrektionsfaktoren. Es haben sich Messgeräte ohne Bedienungseinheiten (sogenannte System-Geräte) herausgebildet, die ausschließlich mit einem externen Rechner für Messaufgaben anzuwenden sind. Für den ständig wachsenden Kreis der Verbraucher ist die VXI- und PXI-Norm von modularen Messgeräten mit der Weiterentwicklung der VME Norm Zustande gebracht. Die Einbettung bedeutet auch, dass in den Computer einbettbare, mit dem Rechner eine Einheit bildende Messgeräte erschienen sind. Die Einbettung der Rechner hat eine nicht zu vernachlässigende Wirkung auf die Instrumente der Mensch-Maschinen-Kopplung: in den Messgeräten sind die im Rechner gebräuchliche Funktionstaste, 
Berührungsbildschirm (Touch-screen), die Menüsteuerung, die Maus, die Klaviatur, sogar der Sprachsynthesizer und die akustische Sprachsteuerung erschienen. Im Messprozess ist die verteilte Intelligenz als grundsätzliche Veränderung zu betrachten: Die Informationen müssen möglichst an der Stelle der Entstehung verarbeitet werden, so müssen nur die Speicherung und Übertragung der besonders wichtigen Informationen realisiert werden. Um die Zuverlässigkeit der Systeme zu erhöhen, entstand die Methode des Logs über die Vorgänge (Log über die externen Vorgänge und über den Eingriff des Administrators). Um den hohen Grad der Kompatibilität der Messgeräte garantieren zu können, wurden aufeinander aufbauende offene Normen (wie IEEE 488.2, SCPI, VPP, VISA, IVI) ausgearbeitet. So sind die Steuerprogramme vom Hersteller des Gerätes oder vom Typ der Schnittstelle immer unabhängiger geworden. Die grafische Programmierung benötigt immer weniger rechentechnische Vorkenntnisse vom Anwender, in dessen Arbeit der Mess-Steuerungs-Algorithmus betont werden kann.

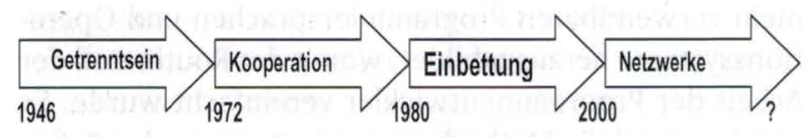

Für die Kooperation der Messgeräte mit den Rechnern ist in der letzten Zeit die Anwendung der Netzwerke typisch. In der Periode vom Jahre 2000 an sind immer mehr Geräte dazu geeignet, direkt an das lokale Netz (LAN) oder an ein Fernnetz (WAN) angeschlossen zu werden. So sind die inneren Zustände zu programmieren bzw. die Messresultate abzufragen. Ein neuer Typ der Steuerungsprogramme beginnt sich herauszubilden: der Browser für Messgeräte, ähnlich den bisherigen Browsers fürs Suchen in Datenbanken. Eine neue Möglichkeit im Laufe des Messprozesses bedeutet die Steuerung und die Datensammlung direkt aus dem Büro.

\section{Die Intelligenz der Messgeräte}

Aufgrund der oben kurz beschriebenen Gesichtspunkte über die Faktoren der menschlichen Intelligenz bzw. aufgrund des ausfuihrlicher analysierten Prozesses der Kopplung zwischen den Geräten und den Rechnern können wir mit Sicherheit bestätigen, dass die Intelligenz der Messgeräte eine wirklich existierende, sich voll entfaltende Erscheinung ist. Der menschlichen Intelligenz ähnlich können die Faktoren der Intelligenz von Messgeräten interpretiert werden, an gewissen Stellen aber scheint es angebracht zu sein, die Begriffe der Psychologie und der Soziologie für die Begriffe der Technik und der Informatik auszutauschen. Das Messgerät versteht die aus der menschlichen Umgebung in Textform kommenden (kodierten, schriftlichen) Hinweise und Informationen, führt eine Tätigkeit dem Inhalt dieser Hinweise und Informationen entsprechend durch und teilt die Ergebnisse der durchgeführten Tätigkeiten in Textform (kodiert, schriftlich) mit. Der Empfang der Informationen aus einer kuinstlichen Umgebung und die Reaktion darauf ist eine Grundfähigkeit der Meßssgeräte. Dieser
Faktor könnte zusammengefasst als erfolgreiche Kommunikation mit der Umgebung formuliert werden.

Die Durchführung der abstrakten arithmetischen und logischen Operationen mit Hilfe eines ins Gerät eingebetteten Rechners ist ein grundsätzlicher IntelligenzFaktor, also im Hinsicht auf Geschwindigkeit und Zuverlässigkeit haben diese Geräte einen Vorteil dem Mensch gegenuiber.

Die Messgeräte sind fähig, solche Parameter der Umgebung wahrzunehmen, die nötig sind, die vorgeschriebenen Aufgaben korrekt auszufuihren. Was die Genauigkeit, Zuverlässigkeit, das Angebot und die Größe der messbaren Mengen betrifft, sind die Messgeräte auf diesem Gebiet dem menschlichen Wahrnehmen gegenuiber im Vorteil. Das Fehlen der Perzeption solcher Parameter, die zu der erfolgreichen Funktion des Gerätes nicht unbedingt nötig sind (z. B. die Farbe und das Gewicht eines Lautsprechers, wenn die Tonqualität und die Bandbreite gemessen werden), dürfen keinesfalls als Negativum betrachtet werden.

Die technischen Bedingungen der Erinnerung werden in den Messgeräten realisiert, nur der Preis kann diesen Bedingungen Schranken setzen. Es gibt schon gewisse Erfolge, wie auf die Erinnerungen assoziativ zurïckgegriffen werden kann, aber die perfekte technische Lösung (und ihre Verbreitung in der Praxis) lässt noch auf sich warten.

Die Feststellung der deterministischen oder stochastischen mathematischen/logischen Beziehungen zwischen den vom Messgerät wahrgenommenen Signalen und den externen Informationen kann zusammenfassend Folgerung genannt werden. Auf diesem Gebiet der Intelligenz haben die Messgeräte einen bedeutenden Nachteil dem Menschen gegenuiber.

Der Vergleich der menschlichen und künstlichen Intelligenz weist auf beiden Seiten Vor- und Nachteile auf. Mit der Entwicklung der Computerwissenschaft kann man mit der Verschiebung der Proportionen rechnen und damit, dass in der Zukunft eine noch weiter entwickelte Generation der Messgeräte mit künstlicher Intelligenz erscheint.

\section{Autor}

\section{Dr. József Kohut}

Technische Hochschule Budapest, Kandó Kálmán

Fakultät für Elektroingenieurwesen

Institut fuir Instrumentation und Automation

1431Budapest, Ungarn

Tavaszmezö ueta 17

E-Mail: kohut@nfs.jozsef.kando.hu 